\title{
Stauntonia hexaphylla (Lardizabalaceae) leaf methanol extract inhibits osteoclastogenesis and bone resorption activity via proteasome- mediated degradation of c-Fos protein and suppression of NFATc1 expression
}

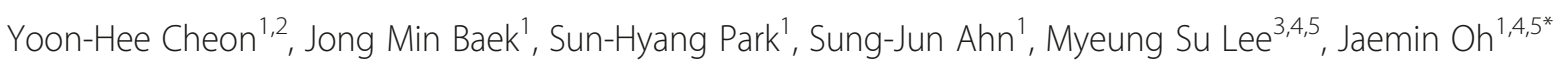
and Ju-Young Kim ${ }^{5^{*}}$

\begin{abstract}
Background: Natural plants, including common vegetables and fruits, have been recognized as essential sources for drug discovery and the development of new, safe, and economical medicaments. Stauntonia hexaphylla (Lardizabalaceae) is widely distributed in Korea, Japan, and China, and is a popular herbal supplement in Korean and Chinese folk medicine owing to its analgesic, sedative, and diuretic properties. However, the exact pharmacological effects of $S$. hexaphylla extract, particularly its effect on osteoclastogenesis, are not known.

Methods: Osteoclast differentiation and function were identified with tartrate-resistant acid phosphatase (TRAP) staining and bone resorption assay, and the underling mechanisms were determined by real-time RT-PCR and western blot analysis.

Results: S. hexaphylla was found to inhibit early-stage receptor activator of nuclear factor-kB (NF-kB) ligand (RANKL)-mediated osteoclast differentiation in bone marrow macrophages (BMMs) without cytotoxicity and bone-resorbing activity in mature osteoclasts in a dose-dependent manner. This $\mathrm{S}$. hexaphylla-mediated blockade of osteoclastogenesis involved abrogation of the NF-KB, ERK, and c-Src-Btk-PLCY2 calcium signal pathways. Interestingly, we found that $\mathrm{S}$. hexaphylla down-regulated RANKL-associated c-Fos protein induction by suppressing its translation. Furthermore, ectopic overexpression of c-Fos and NFATC1 rescued the inhibition of osteoclast differentiation by $S$. hexaphylla. Furthermore, S. hexaphylla inhibited the c-Fos- and NFATc1-regulated expression of genes required for osteoclastogenesis, such as TRAP, OSCAR, $\beta 3$-integrin, ATP6rOd2, and CtsK.
\end{abstract}

Conclusions: These findings suggest that $S$. hexaphylla might be useful for the development of new anti-osteoporosis agents.

Keywords: Stauntonia hexaphylla, Osteoclast, c-Fos, NFATc1, Bone resorption

\footnotetext{
* Correspondence: jmoh@wku.ac.kr; kimjy1014@gmail.com

${ }^{1}$ Department of Anatomy, School of Medicine, Wonkwang University, Iksan, Jeonbuk 570-749, Republic of Korea

${ }^{5}$ Imaging Science based Lung and Bone Diseases Research Center, Wonkwang University, Iksan 570-749, Republic of Korea

Full list of author information is available at the end of the article
} 


\section{Background}

Osteoporosis, characterized by abnormally low bone mineral density and micro-architectural degeneration of bone tissue, is a progressive systemic skeletal disease with various causes, including menopause; advanced age; low calcium and vitamin D intake; and lack of exercise due to long-term bed rest, especially in the elderly population [1-3]. Current anti-osteoporosis drugs such as estrogen, raloxifene, bisphosphonates, and calcitonin have been widely used, and their bone protective mechanisms are based on the inhibition osteoclastic bone resorption. However, these drugs have numerous side effects, causing many patients to discontinue their use [4-7]. Hence, to prevent or reverse osteolytic bone diseases, it is necessary to develop novel agents that have fewer undesirable side effects.

Osteoclasts are specialized, multinucleated, and boneresorbing cells that form part of the mononuclear phagocyte system [1]. They are formed by the fusion of circulating mononuclear precursor cells of hematopoietic origin. Osteoclast differentiation is critically regulated by macrophage colony-stimulating factor (M-CSF) and receptor activator of nuclear factor-kappa B (NF-kB) ligand (RANKL) $[1,8,9]$. M-CSF stimulates the expression of RANK, the receptor for RANKL, and supports the survival and proliferation of osteoclast precursors [9]. RANKL stimulates differentiation of precursors into osteoclasts by inducing the expression of osteoclast-specific genes, including tartrate-resistant acid phosphatase (TRAP), osteoclast-associated receptor (OSCAR), and cathepsin $\mathrm{K}(\mathrm{CtsK})[1,8,10]$. The RANK/RANKL pathway is considered to be an attractive therapeutic target for bone destructive diseases. Although a more detailed mechanism is still expected to be unveiled, the major signaling events have been elucidated: RANK/RANKL binding activates downstream early signaling pathways, including mitogen-activated protein kinase (MAPK) pathways and NF- $\mathrm{KB}$, by recruitment of TNF-receptor associated factor 6 (TRAF6), which regulates transcription factors involved in osteoclast differentiation [11, 12]. Subsequently, RANKL induces the expression of c-Fos and nuclear factor of activated T-cells, cytoplasmic 1 (NFATc1), key transcription factors for osteoclastogenesis [13-15]. NFATc1 is a master regulator of terminal osteoclastogenesis, and autoamplification of NFATc1 depends on activator protein (AP)-1 complex containing c-Fos and NF- $k B$ [15]. In addition, the RANKL/RANK signaling pathway activates $\mathrm{Ca}^{2+}$ signaling through the activation of phospholipase $\mathrm{C} \gamma(\mathrm{PLC} \gamma)[16,17]$. PLCY regulates protein kinase $\mathrm{C}(\mathrm{PKC})$ activation, intracellular $\mathrm{Ca}^{2+}$ levels, and NFATc1 expression in hematopoietic systems $[16,18]$. During osteoclastogenesis, increased $\mathrm{Ca}^{2+}$ levels induce NFATc1 dephosphorylation and NFATc1 translocation into the nucleus. Calcineurin inhibitors, such as FK506 and cyclosporine $\mathrm{A}$, as well as the $\mathrm{Ca}^{2+}$ chelator BAPTA-AM, potently suppress RANKL-induced osteoclastogenesis through inhibition of NFATc1 nuclear translocation [19].

Recently, many plants and their extracts have been recognized as useful sources for the prevention and treatment of bone-related disorders. Stauntonia hexaphylla (Lardizabalaceae) is widely distributed in thickets in lowlands and foothills in warmer regions of Korea, Japan, and China. S. hexaphylla has been used in Chinese folk medicine as analgesics, sedatives, and diuretics [20]. As part of a search for bioactive compounds, methanol extract from the aerial part of $S$. hexaphylla was found to exhibit significant cytotoxic activity and anti-cancer effects on HCT116 human colon cancer cells [21]. However, the exactly pharmacological effect of $S$. hexaphylla remains unknown; in particular, the effect of $S$. hexaphylla on osteoclast differentiation and pathological bone destruction has not yet been well defined. In this study, we have investigated the effects of $S$. hexaphylla on osteoclast differentiation and function, and its possible mechanism of action.

\section{Methods}

\section{Reagents and antibodies}

Methanol extract from the $S$. hexaphylla leaf was purchased from the Korean Plant Extract Bank (Daejeon, Korea). Recombinant soluble human M-CSF and RANKL were obtained from PeproTech EC Ltd. (London, UK). Monoclonal $\beta$-actin antibody was obtained from Sigma (St. Louis, MO, USA). Anti-Akt, anti-phospho-Akt, antip38, anti-phospho-p38, anti-JNK, anti-phospho-JNK, antiphospho-IKB, and anti-phospho-PLC $\gamma 2$ were purchased from Cell signaling Technology Inc. (Beverly, MA, USA). Anti-c-Fos, anti-NFATc1, anti-IkB, and anti-PLC 22 were purchased from Santa Cruz Biotechnology (Santa Cruz, CA, USA). Fetal bovine serum (FBS), $\alpha$-minimum essential medium ( $\alpha$-MEM), and penicillin/streptomycin were purchased from Gibco BRL (Grand Island, NY, USA). All other chemicals were of analytical grade or complied with the standards required for cell culture experiments. Cyclohexamide (CHX), MG132, and Ac-Leu-Leu-nle-h (ALLN) were obtained from Calbiochem (San Diego, CA, USA).

\section{Mice and in vitro osteoclastogenesis assay}

BMMs isolation was performed in accordance with the guidelines for animal experimentation of the Institutional Animal Care and Use Committee of Wonkwang University (WKU15-48, committee member: Sung Yeon Kim, Jungkee Kwon, Hong Geun Oh, Hong-Seob So, Okjin Kim, Chun-Soo Ko). Five-week-old male ICR mice were purchased from Damul Science (Daejeon, Korea). The mice were housed in cages in a controlled 
temperature $\left(22-24{ }^{\circ} \mathrm{C}\right)$ and humidity $(55-60 \%)$ room with a $12 \mathrm{~h}$ light/dark cycle. Bone marrow cells (BMCs) were obtained from 5-week-old male ICR mice by flushing the tibias and femurs with $\alpha$-MEM supplemented with $10 \% \mathrm{FBS}$, penicillin $(100 \mathrm{U} / \mathrm{mL})$, and streptomycin $(100 \mu \mathrm{g} / \mathrm{mL})$. To obtained bone marrow macrophages (BMMs) for osteoclast differentiation of primary BMCs, the cells were cultured in $\alpha$-MEM supplemented with $10 \%$ FBS and M-CSF $(10 \mathrm{ng} / \mathrm{mL})$ for 1 day on culture dishes. Non-adherent cells were transferred to $10 \mathrm{~cm}$ petri dishes and further cultured in the presence of M-CSF (30 ng/mL) for 3 days. Adherent cells were used as BMMs or as osteoclast precursors, after the non-adherent cells were removed. To generate osteoclasts from the BMMs culture system, cells were cultured with M-CSF $(30 \mathrm{ng} / \mathrm{mL})$ and RANKL $(100 \mathrm{ng} / \mathrm{mL})$ in the presence or absence of $S$. hexaphylla on 48-well plates, placed in an incubator set to 37 and $5 \% \mathrm{CO}_{2}$, and allowed to incubate for 3 days. The cells were fixed in $3.7 \%$ formalin for $10 \mathrm{~min}$, permeabilized with $0.1 \%$ Triton X-100, and then stained with TRAP solution. TRAP-positive multinucleated cells (MNCs) with more than three nuclei were counted as osteoclasts.

\section{PCR primers and quantitative real-time RT-PCR}

Oligonucleotides used in this experiment were commercially synthesized by Bioneer Co. (Daejeon, Korea). Real-time RT-PCR analysis was performed using an ExicyclerTM 96 Real-Time Quantitative Thermal Block (Bioneer Co.). The real-time RT-PCR reactions were conducted with initial denaturation for $10 \mathrm{~min}$ at $95{ }^{\circ} \mathrm{C}$, followed by 40 cycles of denaturation for $1 \mathrm{~min}$ at $95^{\circ}$ $\mathrm{C}$, annealing for $30 \mathrm{~s}$ at $60{ }^{\circ} \mathrm{C}$, and extension for $1 \mathrm{~min}$ at $72{ }^{\circ} \mathrm{C}$. The expression of target molecules was analyzed using SYBR Green-based real-time RT-PCR, and normalized to the expression of GAPDH. The primer sets used in the quantitative real-time RT-PCR are shown in Table 1.

Table 1 The oligonucleotides used as primers

\begin{tabular}{lll}
\hline Gene & Forward primer (5'-3') & Reverse primer (5'-3') \\
\hline c-Fos & GGTGAAGACCGTGTCAGGAG & TATTCCGTTCCCTTCGGATT \\
NFATC1 & GAGTACACCTCCAGCACCTT & TATGATGTCGGGGAAAGAGA \\
TRAP & ACTTCCCCAGCCCTTACTACCG & TCAGCACATAGCCCACACCG \\
OSCAR & GGAATGGTCCTCATCTCCTT & TCCAGGCAGTCTCTTCAGTT \\
CtsK & CCAGTGGGAGCTATGGAAGA & CTCCAGGTATGGGCAGAGA \\
B3- & GGAGTGGCTGATCCAGATGT & TCTGACCATCTTCCCTGTCC \\
integrin & & \\
ATPGVOd2 & GACCCTGTGGCACTIITGT & GTGTTGGGCTTGGGAGAA \\
GAPDH & TCAAGAAGGTGGTGAAGCAG & AGTGGGAGTTGCTGTTGAAGT \\
\hline
\end{tabular}

\section{Cytotoxicity assay}

Cell proliferation was measured using the XTT assay kit (Sigma). The BMMs $\left(1 \times 10^{4} /\right.$ well $)$ were seeded in 96 well plates with various concentrations of $S$. hexaphylla and cultured for 3 days in the presence of M-CSF $(30 \mathrm{ng} / \mathrm{mL})$. For the last $4 \mathrm{~h}$ of culture, $50 \mu \mathrm{L}$ of XTT solution was added, and the plate was read at $450 \mathrm{~nm}$ with an ELISA reader (Molecular Devices, CA, USA).

\section{Western blot analysis}

After the specified treatment, cells were washed twice with cold-PBS and lysed with lysis buffer containing $50 \mathrm{mM}$ Tris- $\mathrm{HCl}, 150 \mathrm{mM} \mathrm{NaCl}, 5 \mathrm{mM}$ EDTA, 1 \% Triton $\mathrm{X}-100,1 \mathrm{mM}$ sodium fluoride, $1 \mathrm{mM}$ sodium vanadate, $1 \%$ deoxycholate, and protease inhibitors. The protein concentration was determined using a Bio-Rad DC protein assay kit (Bio-Rad Laboratories., Hercules, CA, USA). The same amount of protein (15-30 $\mu \mathrm{g})$ was separated using $10 \%$ SDS-polyacrylamide gel and transferred to a polyvinylidene difluoride (PVDF, Millipore, Bedford, MA, USA) membrane for $90 \mathrm{~min}$ at $25 \mathrm{~V}$ on an XCell II blot module (Invitrogen, Carlsbad, CA, USA). The PVDF membranes were placed in $5 \%$ nonfat milk in tris-buffered saline with $0.1 \%$ Tween 20 (TBST) for $1 \mathrm{~h}$, washed, and incubated with the primary antibodies for $2 \mathrm{~h}$ at room temperature. The membranes were washed in TBST, and incubated for $1 \mathrm{~h}$ with various HRP-conjugated donkey anti-rabbit, sheep anti-mouse IgG secondary antibodies. The immunoreactive signals were detected using the Western Chemiluminescent HRP substrate kit (Millipore, Billerica, USA).

\section{Bone resorption assay}

Primary osteoblast cells $\left(1 \times 10^{6}\right.$ cells $)$ and BMCs $\left(1 \times 10^{7}\right.$ cells) were cultured on collagen-gel-coated culture dishes for 7 days in the presence of $10^{-8} \mathrm{M}$ 1,25-dihydroxyvitamin D3 (Sigma) and $10^{-6} \mathrm{M}$ prostaglandin $\mathrm{E}_{2}\left(\mathrm{PGE}_{2}\right)$ (Sigma). The co-cultured osteoclasts were detached by $0.1 \%$ collagenase treatment at $37{ }^{\circ} \mathrm{C}$ for $10 \mathrm{~min}$ and then placed on dentine slices or hydroxyapatite coated plates (Corning, NY, USA) with or without S. hexaphylla. After $24 \mathrm{~h}$, the cells were removed, and the total resorption pits were observed under a microscope and then quantified using Image-Pro Plus version 4.0 (Media Cybernetics, Silver Spring, MD, USA).

\section{Retrovirus preparation and infection}

Plat-E retroviral packaging cells were transfected by pMX-IRES-EGFP, pMX-c-Fos-IRES-EGFP, and pMXCA-NFATc1-IRES-EGFP using X-tremeGENE 9 (Roche, Nutley, NJ, USA) according to the manufacturer's protocol. The culture supernatant of the retrovirus-producing cells was collected for retroviral infection on BMMs that were cultured with BMCs in M-CSF (30 ng/mL) for 
2 days. The BMMs were incubated with the retrovirus soup produced by Plat-E cells together with polybrene $(10 \mu \mathrm{g} / \mathrm{mL})$ and M-CSF $(10 \mathrm{ng} / \mathrm{mL})$ for $6 \mathrm{~h}$. Infected BMMs were further cultured in the presence of M-CSF (30 $\mathrm{ng} / \mathrm{mL}$ ) and RANKL (100 $\mathrm{ng} / \mathrm{mL}$ ) with or without $S$. hexaphylla for 3 days. Osteoclast formation was detected by TRAP staining.

\section{c-Fos stability}

The BMMs were incubated with c-Fos retro-virus soup produced by Plat-E cells together with polybrene $(10 \mu \mathrm{g} / \mathrm{mL})$ and M-CSF $(10 \mathrm{ng} / \mathrm{mL})$ for $6 \mathrm{~h}$. Infected BMMs were pretreated with or without $S$. hexaphylla in the presence of M-CSF for $24 \mathrm{~h}$, and then stimulated with RANKL. After 20 h, $2 \mu \mathrm{g} / \mathrm{mL}$ CHX, $5 \mu \mathrm{M}$ MG132, and $20 \mu \mathrm{M}$ ALLN were added to the cultures for $4 \mathrm{~h}$ before harvest.

\section{Statistical analysis}

Experiments were conducted at least three times, and the data are expressed as the mean \pm standard deviation (SD). All statistical analyses were conducted using the Statistical Package for the Social Sciences Software (SPSS; Korean version 14.0). Most of the statistical differences were analyzed using one-way ANOVA followed by Tukey's post hoc test. $P$-values of less than 0.05 were considered statistically significant.

\section{Results}

S. hexaphylla suppresses RANKL-induced osteoclast differentiation in BMMs and bone resorption by co-cultured mature osteoclast

To identify the efficacy of $S$. hexaphylla on RANKLinduced osteoclastogenesis, BMMs were cultured in the presence of RANKL and M-CSF treated with or without various concentrations of $S$. hexaphylla. However, RANKL differentiated the BMMs of the control into TRAPpositive MNCs, and S. hexaphylla dose-dependently decreased the formation of TRAP- positive MNCs (Fig. 1a and b). Next, we evaluated the XTT assay to determine whether the inhibitory effect of S. hexaphylla on osteoclastogenesis was due to reduced viability or proliferation of the osteoclast precursor cells. S. hexaphylla had no cytotoxic effects, did not affect cell proliferation, and inhibited osteoclast differentiation (Fig. 1c). To determine the time course of the inhibitory effect of $S$. hexaphylla on osteoclast differentiation, BMMs were cultured by treatment with $S$. hexaphylla $(50 \mu \mathrm{g} / \mathrm{mL})$ at four different time points with RANKL and M-CSF. The cells incubated for $24 \mathrm{~h}$ in an $S$. hexaphylla-containing medium, and were then transferred to an $S$. hexaphylla-free medium. As shown in Fig. 1d, the time frames in which $S$. hexaphylla most effectively suppressed osteoclast formation were days $0-1$ and 1-2.
However, its inhibitory effect was slightly weaker on days 2-3 and 3-4 (Fig. 1d and e). These finding suggest that $S$. hexaphylla exerts an inhibitory effect on early differentiation. We next examined whether $S$. hexaphylla can reduce the function of mature osteoclasts. Mature osteoclasts were cultured on hydroxyapatitecoated plates in the presence of $S$. hexaphylla. The addition of $S$. hexaphylla clearly inhibited bone resorption activity relative to the DMSO control (Fig. If and $\mathrm{g})$.

\section{S. hexaphylla suppresses RANKL-induced ERK, NF-KB, and $\mathrm{Ca}^{2+}$ signaling pathways}

RANKL binding to RANK results in the recruitment of multiple downstream signaling pathways such as Akt, p38, JNK, ERK, and NF-kB. We examined the effect of S. hexaphylla on RANKL-induced early signaling. As shown in Fig. 2a, RANKL-induced phosphorylation of p38, JNK, and Akt was not affected by $S$. hexaphylla treatment, but $S$. hexaphylla inhibited the phosphorylation of ERK and degradation of IKB. We next examined whether S. hexaphylla alters the RANKL-PLCY2- $\mathrm{Ca}^{2+}$-related signal pathway in which $\mathrm{Ca}^{2+}$ oscillation is followed by promotion of NFATc1. S. hexaphylla completely blocked RANKL-mediated c-Src, Btk, and PLC 2 phosphorylation (Fig. 2a and b).

\section{S. hexaphylla downregulates RANKL-induced expression of NFATc1 and c-Fos stability}

c-Fos and NFATc1 are key transcription factors in RANKL-induced osteoclast differentiation, and they regulate expression of the genes involved in osteoclast differentiation and function such as TRAP, OSCAR, and $C t s K$. Therefore, we investigated $c$-Fos and NFATc1 mRNA expression. As previously reported, c-Fos and NFATc1 expression was upregulated in BMMs by RANKL stimulation. S. hexaphylla decreased the increased expression of the c-Fos protein by RANKL without any marked changes in c-Fos mRNA expression (Fig. 3a and b). Next, we examined whether S. hexaphylla promotes c-Fos protein degradation. After $48 \mathrm{~h}$ post-transfection with c-Fos, the cells treated with CHX, an inhibitor of protein synthesis, decreased c-Fos protein expression compared to control (Fig. 3c); additional S. hexaphylla treatment accelerated this decrease in c-Fos protein levels; and the effect of $S$. hexaphylla was reversed by co-treatment with selective proteasome inhibitors, namely, MG132 or ALLN. These results suggest that proteasomemediated degradation is related to the $S$. hexaphyllainduced reduction in c-Fos protein (Fig. 3c). Next, we examined whether forced expression of c-Fos and NFATc1 was sufficient to reverse the inhibitory effect of $S$. hexaphylla on osteoclast differentiation. The 
a

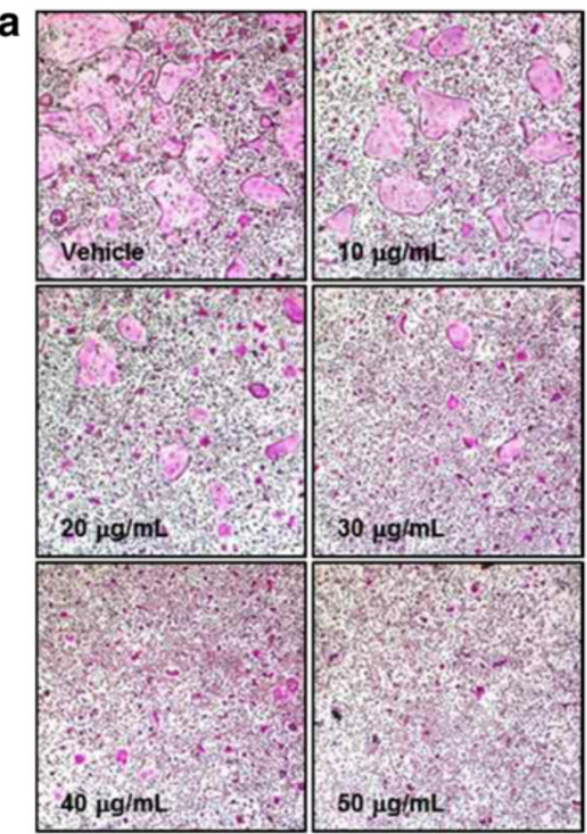

d

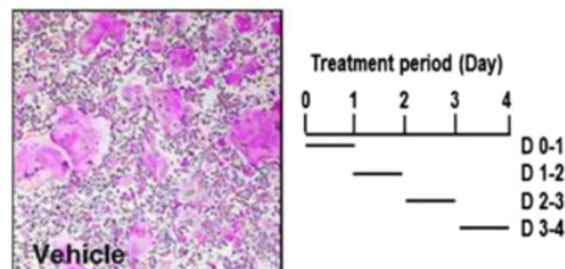

b

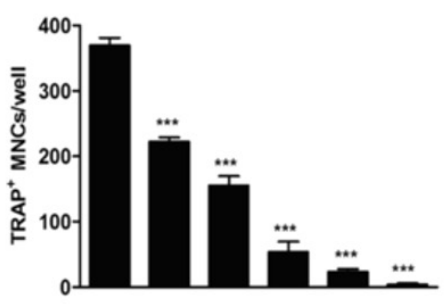

C

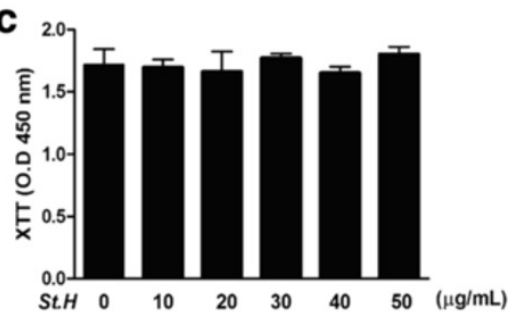

e 500

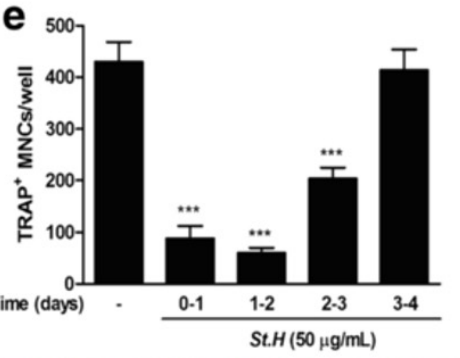

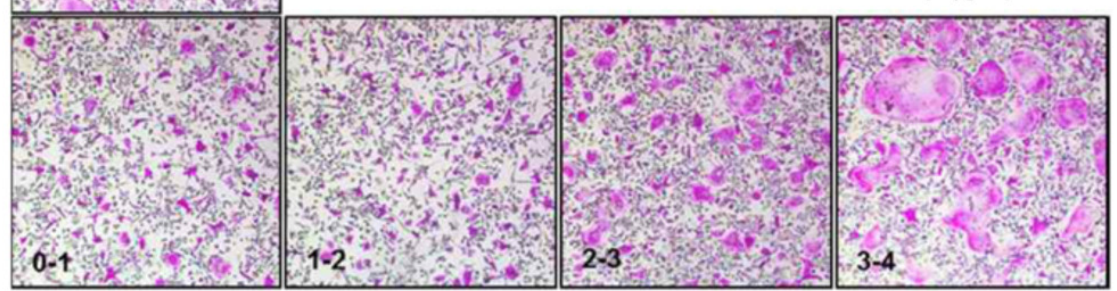

f

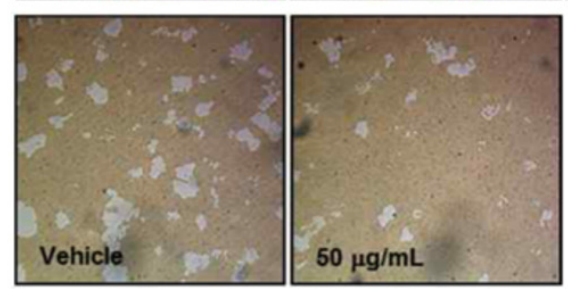

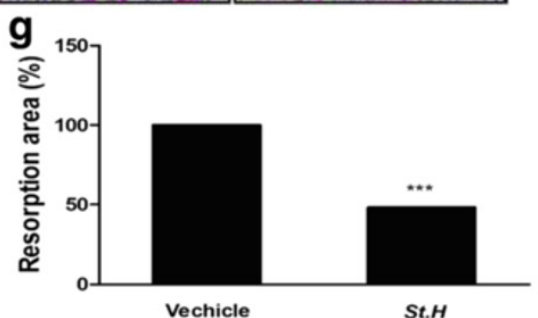

Fig. 1 S. hexaphylla suppresses RANKL-induced osteoclast differentiation in BMMs and bone resorption by co-cultured mature osteoclasts. a BMMs were cultured with M-CSF $(30 \mathrm{ng} / \mathrm{mL})$ and RANKL $(100 \mathrm{ng} / \mathrm{mL})$ for 3 days in the presence or absence of $S$. hexaphylla. b After culturing, cells were fixed with $3.7 \%$ formalin in PBS, permeabilized with $0.1 \%$ Triton X-100 in PBS, and stained with TRAP staining solution; TRAP-positive MNCs were then counted. c BMMs were cultured for 3 days with the indicated doses of S. hexaphylla in the presence of M-CSF ( $30 \mathrm{ng} / \mathrm{mL}$ ). Cell viability was analyzed using an XTT assay. $\mathbf{d}$ BMMs were cultured as in (a), except that the cells were treated with $\mathbf{S}$. hexaphylla on the indicated days. e After culturing, cells were fixed, and the number of TRAP-positive MNCs was counted. $\mathbf{f}$ pOBs and BMCs were co-cultured on a collagen-matrix-coated plate for 6 days, and the flourishing osteoclasts were harvested. Mature osteoclasts were seeded on hydroxyapatite-coated plates with or without S. hexaphylla for $24 \mathrm{~h}$. Attached cells on the plates were removed and photographed under a light microscope. Pit areas were quantified using Image J (g), and bone resorption numbers were counted using a light microscope. ${ }^{* * *} P<0.001$; versus vehicle (DMSO)

ectopic expression of c-Fos and NFATc1 fully reversed the inhibitory effect of $S$. hexaphylla on RANKLinduced osteoclast differentiation (Fig. 3d-f). These results indicate that the effect of $S$. hexaphylla may involve the inhibition of c-Fos and NFATc1 during RANKL-mediated osteoclastogenesis. 
a
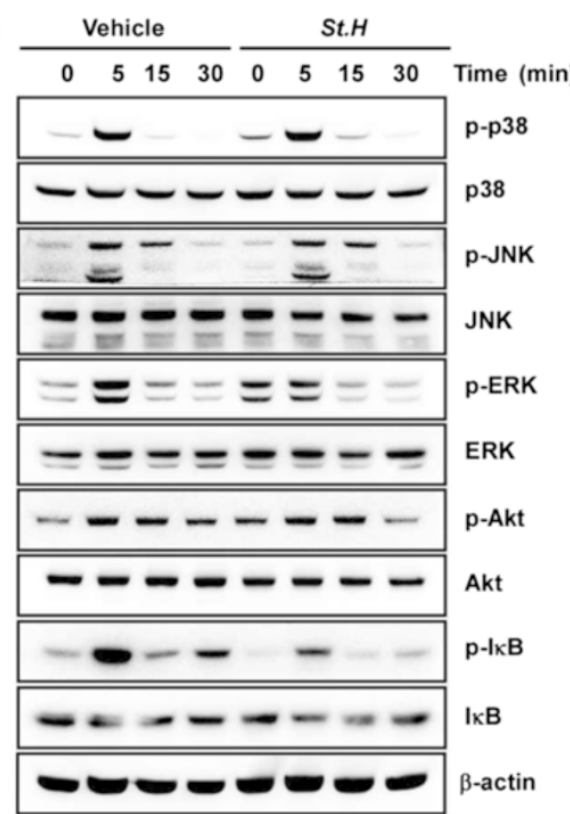

b Vehicle St.H

$\begin{array}{llllllllll}0 & 5 & 15 & 30 & 0 & 5 & 15 & 30 & \text { Time (min) }\end{array}$

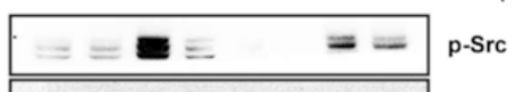

$----\infty$ src

$-\ldots-\ldots-$ p.Btk

$+\ldots-\ldots+\ldots$ Btk

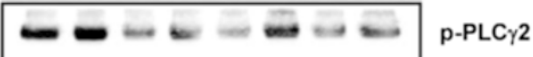

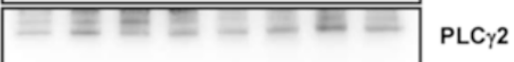

$----\infty-a$-actin

Fig. 2 S. hexaphylla suppresses RANKL-induced ERK, NF-KB, and $\mathrm{Ca}^{2+}$ signaling pathways. BMMs were starved with serum-free a-MEM media for $3 \mathrm{~h}$. BMMs were pretreated with S. hexaphylla for $1 \mathrm{~h}$ and then stimulated with RANKL for the indicated times. BMMs were pretreated with or without S. hexaphylla for $1 \mathrm{~h}$ and then stimulated with RANKL $(100 \mathrm{ng} / \mathrm{mL})$ for the indicated time. The cell lysates were analyzed by western blotting with antibodies. $\beta$-actin was used as the internal control

\section{S. hexaphylla inhibits RANKL-induced mRNA expression of} OSCAR, TRAP, ATP6vOd2, $\beta 3$-integrin, and CtsK

NFATc1 regulates the expression of OSCAR, TRAP, ATP6vod2, B3-integrin, and CtsK during RANKLinduced osteoclastogenesis. We determined whether $S$. hexaphylla regulates the expression of OSCAR, TRAP, ATP6vod2, B3-integrin, and CtsK. S. hexaphylla downregulated the expression of OSCAR and TRAP, which are genes related to osteoclast formation. S. hexaphylla also decreased the expression of ATP6vod2 and B3-integrin, which affect cell-to-cell migration or fusion. The expression of CtsK and its associated bone-resorbing activity was inhibited by S. hexaphylla (Fig. 4), suggesting that the inhibitory effect of $S$. hexaphylla on RANKL-mediated NFATc1 expression is followed by the down-regulation of osteoclastogenic marker genes.

\section{Discussion}

There is a growing interest in the efficacy of natural plant products for the prevention and treatment of bone-related disorders such as osteoporosis and rheumatoid arthritis. In this study, we have shown that $S$. hexaphylla effectively inhibits RANKL-induced osteoclast differentiation at the early stages without any cytotoxicity or bone-resorbing activity by mature osteoclasts (Fig. 1).

RANK/RANKL interaction forms a fundamental cytokine system that is capable of influencing all aspects of osteoclast differentiation, function, and (indirectly) the complete bone regulatory system $[10,12]$.
The connection between RANK and RANKL induces recruitment and activation of TRAF6, which leads to multiple downstream cascades. The signals are composed of six known signal pathways: ERK, p38, JNK, NF-kB, Src, and Akt. We found that by suppressing RANKL-mediated ERK and NF-kB signaling, $S$. hexaphylla inhibited osteoclast differentiation (Fig. 2a). In previous studies, compared to WT mice, ERK1 deficiency reduced the number of osteoclast progenitors and the formation of TRAP-positive cells on the trabecular surface [22]. In addition, NF- $\mathrm{kB}$ is an important signal mediator of inflammatory and immune reactions, and is a major transcription factor for RANKL-activated osteoclastogenesis [23]. IкB is bound to NF- $\mathrm{kB}$, which prevents it from translocating to the nucleus, and phosphorylation by IKK separates the two proteins. Subsequent ubiquitination and proteasomal degradation of I $\mathrm{KB}$ allows NF- $\mathrm{KB}$ to translocate into the nucleus and stimulate the transcription of the target gene [24]. NF-kB p50/p52 double knockout mice exhibit osteoclastogenesis defects and severe osteopetrosis [25, 26]. $S$. hexaphylla also induces osteoclastogenesis through the c-Src-Btk-PLC 2 signaling pathway (Fig. 2b). c-Src deficiency produces an osteopetrotic skeletal phenotype and affects the bone-resorbing activity of mature osteoclasts [27, 28]. PLC $\gamma$ activates $\mathrm{Ca}^{2+}$ signaling and NFATc1 in immune cells. PLC $\gamma 2$ deficiency in mouse cells lowers the expression of NFATc1 RANKLstimulation relative to wild mice [29]. 


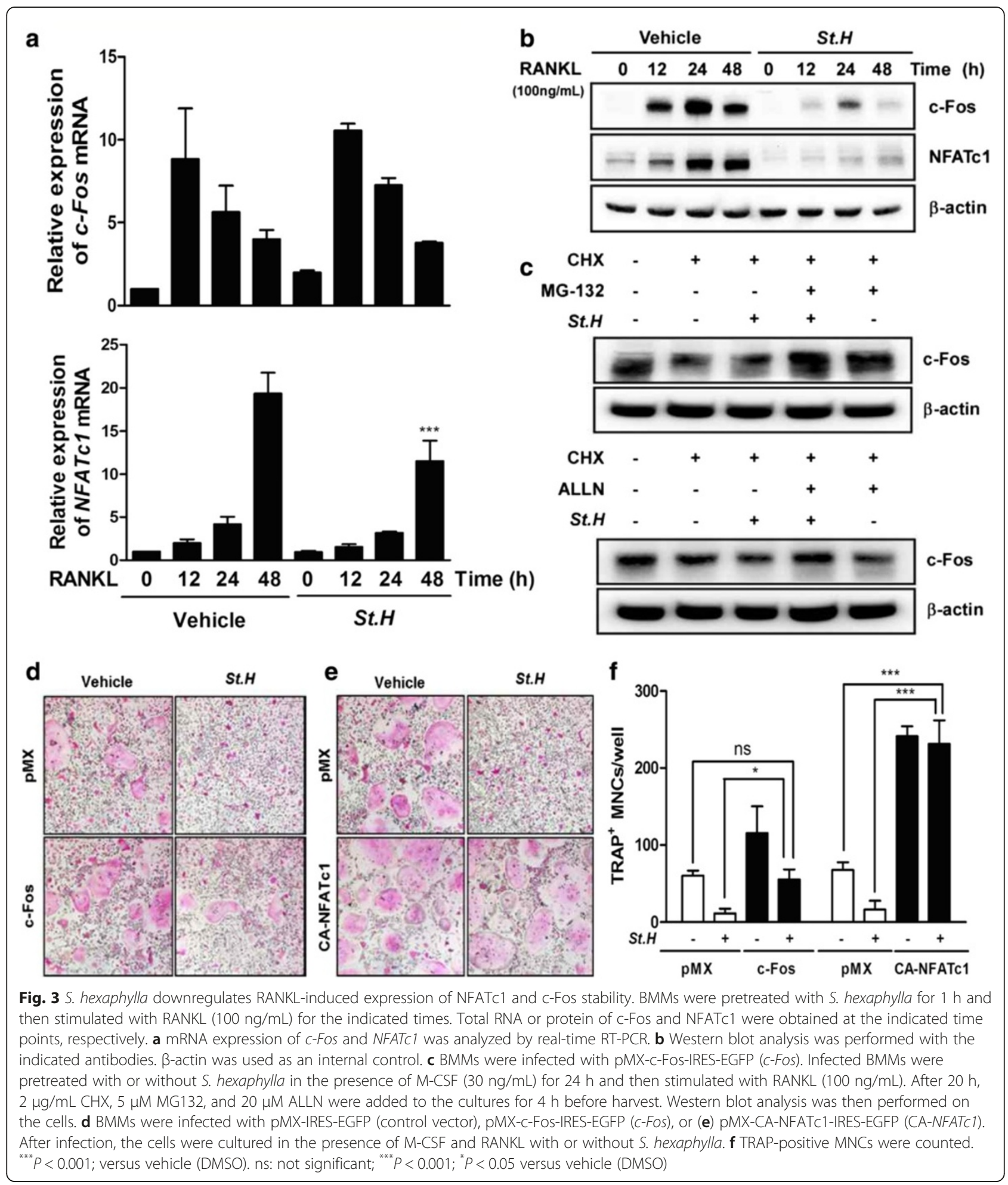

Under normal conditions, the RANKL-RANK axis appears to be essential for osteoclastogenesis, and costimulatory immunoreceptors lead to robust induction of c-Fos and NFATc1, which are the necessary and sufficient transcription factors for osteoclast differentiation. The crucial role of c-Fos in osteoclastogenesis was demonstrated by in vivo experiments using genetically modified mice. c-Fos-deficient mice exhibit a severe osteoporotic phenotype due to the failure of osteoclast differentiation [30]. NFATc1-deficient embryonic stem 

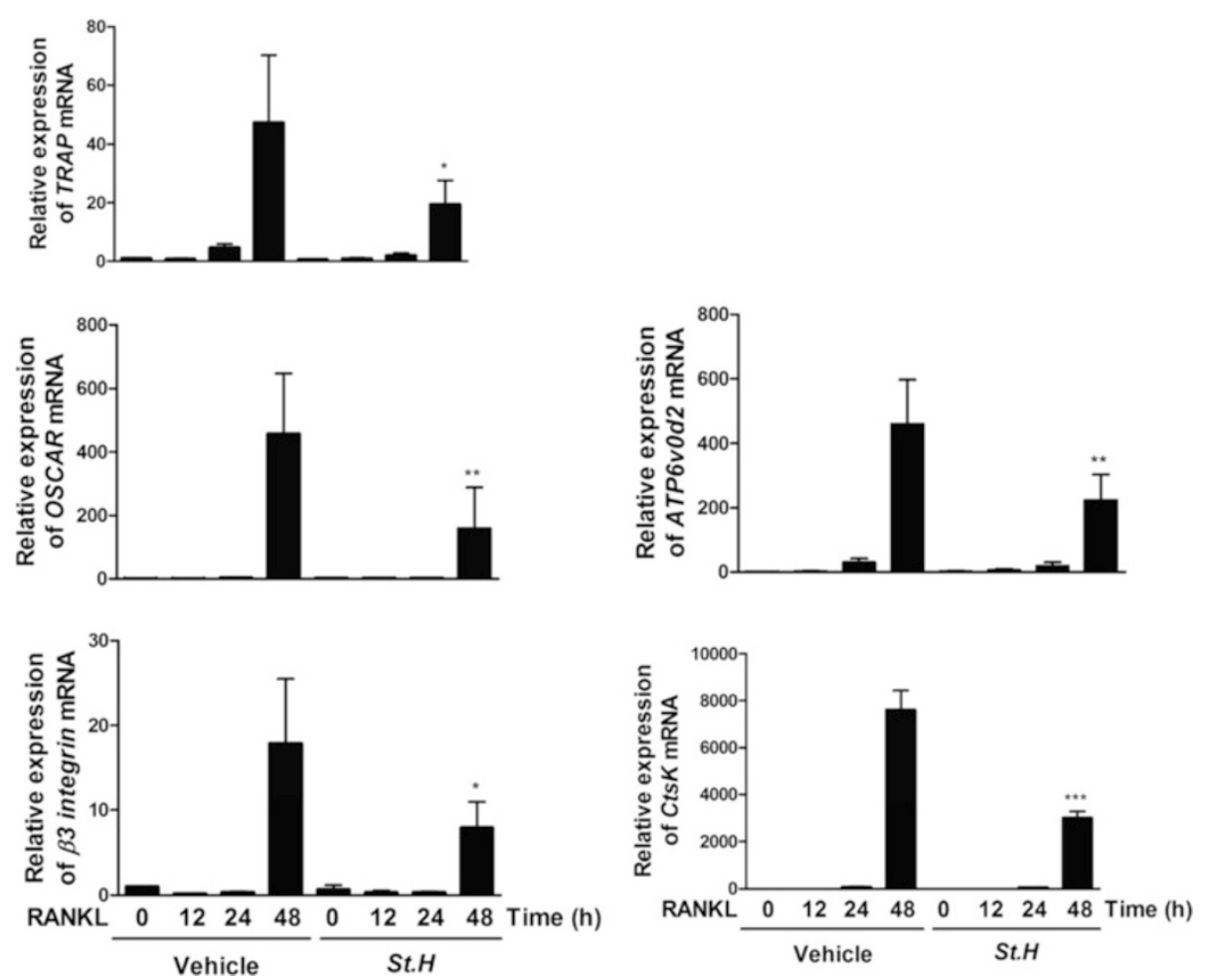

Fig. 4 S. hexaphylla inhibits RANKL-induced mRNA expression of OSCAR, TRAP, ATP6vOd2, $\beta 3$-integrin, and CtsK. BMMs were pretreated with S. hexaphylla for $1 \mathrm{~h}$ and then stimulated with RANKL $(100 \mathrm{ng} / \mathrm{mL})$ for the indicated times. Total RNA was obtained at the indicated time points, respectively. The mRNA expression of OSCAR, TRAP, ATP6VOd2, $\beta 3$-integrin, and CtsK was analyzed by real-time RT-PCR. ns: not significant; ${ }^{* * *} P<$ $0.001 ;{ }^{* *} P<0.01 ;{ }^{*} P<0.05$ versus vehicle (DMSO). ${ }^{* * *} P<0.001 ;{ }^{* *} P<0.01 ;{ }^{*} P<0.05$ versus vehicle (DMSO)

cells are unable to differentiate into osteoclasts in response to RANKL, and the forced expression of NFATc1 leads to the formation of osteoclasts from BMMs in the absence of RANKL [15]. In our study, S. hexaphylla suppressed RANKL-induced NFATc1 mRNA and protein expression. By contrast, c-Fos protein, but not mRNA, expression was diminished (Fig. 3a and b). This suggests that $S$. hexaphylla-mediated inhibition of NFATc1 expression is not involved in c-Fos transcriptional activity, and instead results from the inhibition of c-Fos translational activity. Moreover, the protein stability assay showed that the reduction in cFos protein contributed to the suppression of c-Fos translation via proteasome-dependent degradation (Fig. 3c). Furthermore, the forced expression of $c$-Fos or CA-NFATc1 rescued the $S$. hexaphylla-induced inhibition of osteoclast differentiation, which suggests that downregulation of c-Fos is responsible for the inhibitory effects of S. hexaphylla (Fig. 3d-f).

NFATc1 has a crucial role in the regulation of osteoclastogenic marker genes during RANKL-mediated osteoclast differentiation, and it gradually induces the expression of osteoclast-specific genes, including OSCAR, TRAP, ATP6v0d2, B3-integrin, and CtsK [1, 8, 9]. The present data suggest that $S$. hexaphylla suppressed the induction of OSCAR, TRAP, ATPGVOd2, B3-integrin, and
CtsK (Fig. 4). Several proteolytic enzymes, including OSCAR, TRAP, ATP6v0d2, and CtsK, have been shown to play important roles in degrading the organic bone matrix, and of these proteolytic enzymes have the highest levels in osteoclasts. Matrix-degrading enzymes are also known to be collagen-degrading enzymes, and can directly degrade collagen in hard tissues that have been demineralized. In addition, $\alpha v \beta 3$-integrin is known to play a role in the regulation of cell migration and the maintenance of the sealing zone required for effective osteoclastic bone resorption. This indicates that $S$. hexaphylla extract inhibited bone resorption by disrupting the induction of cytoskeletal organization, leading to the formation of a ruffled border and the adhesion of osteoclasts to the bone matrix.

\section{Conclusions}

In conclusion, this is the first study to report that $S$. hexaphylla efficiently suppresses RANKL-induced osteoclast differentiation and bone-resorbing activity in vitro. S. hexaphylla inhibits osteoclastogenesis via proteasomemediated degradation of c-Fos protein and suppression of NFATc1 induction. These results suggest that $S$. hexaphylla has potential as a natural herbal therapy for diseases associated with bone loss. 


\section{Competing interests}

The authors declare that they have no competing interests.

\section{Authors' contributions}

YHC, JO, JYK: Conception and design, carried out the cell culture and assay, analysis and interpretation of data; draft the manuscript and revise it critically for important intellectual content; final approval of the version to be published; JMB: Carried out the western blot analysis, draft the manuscript; SHP, SJA: Carried out the bone resorption assay and real-time RT-PCR, and interpretation of data; MSL: Conception and design, final approval of the version to be published. All authors read and approved the final manuscript.

\section{Acknowledgements}

This study was supported by a grant from Basic Science Research Program through the National Research Foundation of Korea (NRF) funded by the Ministry of Education (NRF-2013R1A1A2059831).

\section{Author details}

${ }^{1}$ Department of Anatomy, School of Medicine, Wonkwang University, Iksan, Jeonbuk 570-749, Republic of Korea. ${ }^{2}$ Center for Metabolic Function Regulation, Wonkwang University, Iksan, Jeonbuk 570-749, Republic of Korea. ${ }^{3}$ Division of Rheumatology, Wonkwang University, Iksan, Jeonbuk 570-749, Republic of Korea. ${ }^{4}$ Institute for Skeletal Disease, Wonkwang University, Iksan, Jeonbuk 570-749, Republic of Korea. ${ }^{5}$ Imaging Science based Lung and Bone Diseases Research Center, Wonkwang University, Iksan 570-749, Republic of Korea.

\section{Received: 2 June 2015 Accepted: 3 August 2015}

\section{Published online: 14 August 2015}

\section{References}

1. Boyle WJ, Simonet WS, Lacey DL. Osteoclast differentiation and activation. Nature. 2003;423(6937):337-42.

2. Raisz LG. Pathogenesis of osteoporosis. concepts, conflicts, and prospects. J Clin Invest. 2005;115(12):3318-25.

3. Teitelbaum SL, Ross FP. Genetic regulation of osteoclast development and function. Nat Rev Genet. 2003;4(8):638-49.

4. Body JJ. Calcitonin for the long-term prevention and treatment of postmenopausal osteoporosis. Bone. 2002;30(5 Suppl):75S-9S.

5. Eriksen EF, Diez-Perez A, Boonen S. Update on long-term treatment with bisphosphonates for postmenopausal osteoporosis: a systematic review. Bone. 2014;58:126-35.

6. Rodan GA, Martin TJ. Therapeutic approaches to bone diseases. Science. 2000;289(5484):1508-14.

7. Sipos W, Pietschmann P, Rauner M. Strategies for novel therapeutic approaches targeting cytokines and signaling pathways of osteoclasto- and osteoblastogenesis in the fight against immune-mediated bone and joint diseases. Curr Med Chem. 2008;15(2):127-36.

8. Tanaka S, Nakamura K, Takahasi N, Suda T. Role of RANKL in physiological and pathological bone resorption and therapeutics targeting the RANKL-RANK signaling system. Immunol Rev. 2005;208:30-49.

9. Arai F, Miyamoto T, Ohneda O, Inada T, Sudo T, Brasel K, et al. Commitment and differentiation of osteoclast precursor cells by the sequential expression of c-Fms and receptor activator of nuclear factor kappaB (RANK) receptors. J Exp Med. 1999;190(12):1741-54.

10. Zaidi M, Blair HC, Moonga BS, Abe E, Huang CL. Osteoclastogenesis, bone resorption, and osteoclast-based therapeutics. J Bone Miner Res. 2003;18(4):599-609.

11. Kobayashi N, Kadono Y, Naito A, Matsumoto K, Yamamoto T, Tanaka S, et al. Segregation of TRAF6-mediated signaling pathways clarifies its role in osteoclastogenesis. EMBO J. 2001;20(6):1271-80.

12. Takayanagi H, Kim S, Taniguchi T. Signaling crosstalk between RANKL and interferons in osteoclast differentiation. Arthritis Res. 2002;4 Suppl 3:S227-32.

13. Asagiri M, Sato K, Usami T, Ochi S, Nishina H, Yoshida H, et al. Autoamplification of NFATC1 expression determines its essential role in bone homeostasis. J Exp Med. 2005;202(9):1261-9.

14. Grigoriadis AE, Wang ZQ, Cecchini MG, Hofstetter W, Felix R, Fleisch HA, et al. c-Fos: a key regulator of osteoclast-macrophage lineage determination and bone remodeling. Science. 1994;266(5184):443-8.

15. Takayanagi H, Kim S, Koga T, Nishina H, Isshiki M, Yoshida H, et al. Induction and activation of the transcription factor NFATC1 (NFAT2) integrate RANKL signaling in terminal differentiation of osteoclasts. Dev Cell. 2002;3(6):889-901.
16. Faccio R, Cremasco V. PLCgamma2: where bone and immune cells find their common ground. Ann N Y Acad Sci. 2010;1192:124-30.

17. Kertesz Z, Gyori D, Kormendi S, Fekete T, Kis-Toth K, Jakus Z, et al. Phospholipase Cgamma2 is required for basal but not oestrogen deficiency-induced bone resorption. Eur J Clin Invest. 2012;42(1):49-60

18. Patterson RL, van Rossum DB, Nikolaidis N, Gill DL, Snyder SH. Phospholipase C-gamma: diverse roles in receptor-mediated calcium signaling. Trends Biochem Sci. 2005;30(12):688-97.

19. Negishi-Koga T, Takayanagi H. Ca2+-NFATc1 signaling is an essential axis of osteoclast differentiation. Immunol Rev. 2009;231(1):241-56.

20. Wang HB, Yu DQ, Liang XT, Watanabe N, Tamai M, Omura S. Structures of two nortriterpenoid saponins from Stauntonia chinensis. J Nat Prod. 1990;53(2):313-8.

21. Chen Z, Wen L, Martin M, Hsu CY, Fang L, Lin FM, et al. Oxidative stress activates endothelial innate immunity via sterol regulatory element binding protein 2 (SREBP2) transactivation of microRNA-92a. Circulation. 2015;131(9):805-14.

22. He Y, Staser K, Rhodes SD, Liu Y, Wu X, Park SJ, et al. Erk1 positively regulates osteoclast differentiation and bone resorptive activity. PLOS One. 2011;6(9), e24780.

23. Jimi E, Aoki K, Saito H, D'Acquisto F, May MJ, Nakamura I, et al. Selective inhibition of NF-kappa B blocks osteoclastogenesis and prevents inflammatory bone destruction in vivo. Nat Med. 2004;10(6):617-24.

24. Hayden MS, Ghosh S. Signaling to NF-kappaB. Genes Dev. 2004;18(18):2195-224.

25. Zheng $H$, Yu X, Collin-Osdoby P, Osdoby P. RANKL stimulates inducible nitric-oxide synthase expression and nitric oxide production in developing osteoclasts. An autocrine negative feedback mechanism triggered by RANKL-induced interferon-beta via NF-kappaB that restrains osteoclastogenesis and bone resorption. J Biol Chem. 2006;281(23):15809-20.

26. Yamashita T, Yao Z, Li F, Zhang Q, Badell IR, Schwarz EM, et al. NF-kappaB p50 and p52 regulate receptor activator of NF-kappaB ligand (RANKL) and tumor necrosis factor-induced osteoclast precursor differentiation by activating c-Fos and NFATc1. J Biol Chem. 2007;282(25):18245-53.

27. Boyce BF, Yoneda T, Lowe C, Soriano P, Mundy GR. Requirement of pp60c-src expression for osteoclasts to form ruffled borders and resorb bone in mice. J Clin Invest. 1992;90(4):1622-7.

28. Soriano P, Montgomery C, Geske R, Bradley A. Targeted disruption of the c-src proto-oncogene leads to osteopetrosis in mice. Cell. 1991;64(4):693-702.

29. Mao D, Epple H, Uthgenannt B, Novack DV, Faccio R. PLCgamma2 regulates osteoclastogenesis via its interaction with ITAM proteins and GAB2. J Clin Invest. 2006;116(11):2869-79.

30. Arai A, Mizoguchi T, Harada S, Kobayashi Y, Nakamichi Y, Yasuda H, et al. Fos plays an essential role in the upregulation of RANK expression in osteoclast precursors within the bone microenvironment. J Cell Sci. 2012;125(Pt 12):2910-7.

\section{Submit your next manuscript to BioMed Central and take full advantage of:}

- Convenient online submission

- Thorough peer review

- No space constraints or color figure charges

- Immediate publication on acceptance

- Inclusion in PubMed, CAS, Scopus and Google Scholar

- Research which is freely available for redistribution 